\title{
Evaluation and comparison of the potential of two ferritins as anti-tick vaccines against Haemaphysalis longicornis
}

Remil Linggatong Galay ${ }^{1,2}$, Takeshi Miyata ${ }^{3}$, Rika Umemiya-Shirafujii ${ }^{4}$, Hiroki Maeda ${ }^{1,2}$, Kodai Kusakisako 1,2, Naotoshi Tsuji ${ }^{5}$, Masami Mochizuki ${ }^{1,2}$, Kozo Fujisaki $^{6}$ and Tetsuya Tanaka ${ }^{1,2^{*}}$

\begin{abstract}
Background: Tick control is an essential aspect of controlling the spread of tick-borne diseases affecting humans and animals, but it presently faces several challenges. Development of an anti-tick vaccine is aimed at designing cost-effective and environmentally friendly protection against ticks and tick-borne diseases as an alternative to the use of chemical acaricides. A single vaccine from the tick midgut protein Bm86 is currently available for field applications, but its efficacy is limited to only some tick species. Identification of candidate vaccine antigens that can affect multiple tick species is highly desirable. The hard tick Haemaphysalis longicornis has two kinds of the iron-binding protein ferritin (HIFER), an intracellular HIFER1 and a secretory HIFER2, and RNA interference experiments showed that these are physiologically important in blood feeding and reproduction and in protection against oxidative stress. Here we investigated the potential of targeting HIFERs for tick control by immunizing the host with recombinant HIFERs (rHIFER1 and rHIFER2).
\end{abstract}

Methods: Rabbits were immunized with rHIFERs three times subcutaneously at two-week intervals. Antisera were collected before the first immunization and a week after each immunization to confirm the antigen-specific serum antibody titer by serum ELISA. Two weeks after the final immunization, the rabbits were challenged with tick infestation. After dropping, tick feeding and reproduction parameters were evaluated to determine vaccine efficacy. To demonstrate the effects of antibodies, oxidative stress was detected in the eggs and larvae.

Results: The antibody titer of rHIFER-immunized rabbits greatly increased after the second immunization. Antibodies exhibited cross-reactivity with rHIFERs and reacted with tick native HIFERs in Western blot analysis. Significantly lower bodyweight was observed in the ticks infested from the rHIFER2-immunized rabbit compared to those from the control rabbit. Reduced oviposition and hatching rate were observed in both rHIFER-immunized groups. rHIFER2 showed a higher vaccine efficacy. The antibodies against rHIFERs were detected in the eggs, and higher levels of oxidative stress biomarkers in the eggs and larvae, of ticks from rHIFER vaccinated rabbits.

Conclusion: Collectively, these results showed that HIFER2 has a good potential as an anti-tick vaccine antigen that may affect multiple tick species.

Keywords: Ticks, Haemaphysalis longicornis, Ferritin, Vaccine, Iron metabolism, Oxidative stress

\footnotetext{
* Correspondence: tetsuya@ms.kagoshima-u.ac.jp

'Department of Pathological and Preventive Veterinary Science, The United

Graduate School of Veterinary Science, Yamaguchi University, Yoshida,

Yamaguchi 753-8515, Japan

${ }^{2}$ Laboratory of Infectious Diseases, Joint Faculty of Veterinary Medicine,

Kagoshima University, 1-21-24 Korimoto, Kagoshima 890-0065, Japan

Full list of author information is available at the end of the article
} 


\section{Background}

Ticks and tick-borne diseases remain threats to human and animal health worldwide. Aside from the direct damage that ticks inflict on their host, they serve as vectors of a wide variety of pathogens, including protozoa, rickettsiae and viruses. In humans, Lyme borreliosis and tick-borne encephalitis are among the most important diseases transmitted by ticks [1]. In cattle, ticks are responsible for the spread and persistence of theileriosis, anaplasmosis, cowdriosis, and babesiosis [2]. The hard tick Haemaphysalis longicornis, mainly distributed in East Asia and Australia, is a known vector of babesiosis caused by Babesia ovata, $B$. major, B. gibsoni, and possibly B. bigemina and of theileriosis caused by Theileria sergenti, T. orientalis, and T. buffeli $[3,4]$. Recently, $H$. longicornis has been strongly implicated as a vector of severe fever with thrombocytopenia syndrome (SFTS) virus affecting humans, which has been reported in China [5], Japan [6] and South Korea [7].

Effective tick control is essential in preventing tick infestation and, subsequently, the spread of tick-borne pathogens. Until now, the use of chemical acaricides was the primary measure of controlling ticks worldwide. However, concerns about limited efficacy, the emergence of resistant ticks, and contamination of the environment and animal products are among the disadvantages of acaricide application. Vaccination is a promising control alternative that will avoid the drawbacks of acaricide application [8]. Ideally, these vaccines should reduce tick infestation and pathogen transmission [9]. For about 20 years, the only commercially available anti-tick vaccine has utilized the midgut protein Bm86 from Rhipicephalus (Boophilus) microplus as the antigen [10]; however, it is only effective against a limited number of tick species [8]. Although numerous antigens have been studied as candidates for a tick vaccine, no other tick vaccine has progressed to commercial development [11]. The main challenge in anti-tick vaccine development is the identification of a suitable tick protective antigen that can be effective against all developmental stages and a wide range of tick species. Many studies on ticks are now focused on the identification of antigens using combined approaches [12] aimed at targeting multiple tick species and multiple tick-borne pathogens at the same time $[1,13,14]$.

Ferritin (FER) is generally an iron-binding protein consisting of 24 subunits folded in a helical bundle involved in iron homeostasis in most organisms [15]. Two types of ferritin, an intracellular (FER1) and a secretory type (FER2), have been characterized in the hard ticks Ixodes ricinus [16] and $H$. longicornis [17]. These molecules were found to be crucial in the blood feeding and reproduction of these hard ticks. Knockdown experiments through RNA interference (RNAi) in both studies resulted in reduced blood feeding capacity, high mortality after blood feeding, and reduced fecundity $[16,17]$ as consequences of iron overload and oxidative stress [18]. These results implied that FERs of the hard ticks may be good target molecules for tick control. Hajdusek et al. [19] performed vaccination studies using recombinant FER2 against $I$. ricinus and Rhipicephalus microplus. Here we compared the potential of two recombinant FERs of $H$. longicornis, rHIFER1 and rHIFER2, as vaccines for tick control. We also attempted to demonstrate how vaccination using these rHIFERs can affect ticks by examining whether induced antibodies can block the function of native HlFERs.

\section{Methods}

\section{Ticks and animals}

Adult parthenogenetic (Okayama strain) H. longicornis ticks were used for the infestation challenge following host immunization. These ticks were maintained by feeding on the ears of Japanese white rabbits (Kyudo, Kumamoto, Japan) for several generations at the Laboratory of Infectious Diseases, Joint Faculty of Veterinary Medicine, Kagoshima University, Kagoshima, Japan [20]. Rabbits were also used for the whole immunization experiment. The animals were maintained and the experiments performed according to the approved guidelines from Animal Care and Use Committee of Kagoshima University (approval number VM13007).

\section{Expression and purification of recombinant ferritins}

The open reading frame (ORF) of Hlfer1 (GenBank: AY277905) or Hlfer2 (GenBank: AB734098) was extracted from their respective pGCAP1 vector using the following primer sets with $\mathrm{Bam} \mathrm{HI}$ recognition sites: Hlfer1-F 5'ACGGATCCAAAATGGCCGCTACT-3' and Hlfer1-R 5'-ACGGATCCTCCTCAGTCGTCTCC-3' for rHIFER1 or Hlfer2-F 5'-ACGGATCCACCATGCTCCCGATC-3' and Hlfer2-R 5' -ACGGATCCGGTTTATTTGTCGCT-3' for rHIFER2. After cutting with $B a m H I$, the amplified DNA fragments were purified using GENECLEAN II kit (MP Biomedicals LLC, Solon, OH, USA) and then subcloned into the BamHI cutting site of pRSET A vector (Invitrogen, Carlsbad, CA, USA). The constructs, pRSETA/HIFER1 and pRSETA/HIFER2, were expressed in $E$. coli BL21 (DE3) cells grown in Luria-Bertani broth medium with ampicillin. The expression of histidine (His)-tagged rHlFERs was induced with $1 \mathrm{mM}$ final concentration of isopropyl $\beta$-D-1-thiogalactopyranoside (IPTG). After overnight induction, cells were collected by centrifugation at $3,350 \times g$ for $30 \mathrm{~min}$, and the proteins were extracted through ultrasonication. Purification was done through affinity chromatography using a His-trap ${ }^{\text {mM }}$ FF column (GE Healthcare, Uppsala, Sweden) at denatured condition with $6 \mathrm{M}$ urea, followed by dialysis, first against phosphate-buffered saline (PBS) containing $0.5 \mathrm{M}$ arginine for refolding overnight, and then against PBS alone 
overnight. The purity of the rHIFERs was confirmed by SDS-PAGE, and the concentration was determined through SDS-PAGE using bovine serum albumin as the standard and Micro BCA Assay kit (Thermo Scientific, Rockford, IL, USA). The rHIFERs were kept at $-30^{\circ} \mathrm{C}$ until use.

\section{Rabbit immunization}

A total of three rabbits from each group were used for two separate vaccination trials. For each immunization, rHIFER1 or rHIFER2 was thoroughly mixed with an equal volume of incomplete Freund's adjuvant (Sigma-Aldrich, St. Louis, MO, USA) to a final concentration of $100 \mu \mathrm{g}$ per $1.5 \mathrm{~mL}$. The mixture was administered subcutaneously using a sterilized glass syringe and a $21 \mathrm{G}$ needle and repeated three times at two-week intervals. Control rabbits were immunized with adjuvant alone. Sera were collected before the first immunization and a week after each immunization for confirmation of antibody titer (days $0,7,21$, and 35 ).

\section{Measurement of serum antibody levels through ELISA}

The antigen-specific serum antibody titer was determined by ELISA. ELISA plates (F96 Maxisorp, Nunc, Roskide, Denmark) were coated with either rHIFER1 or rHIFER2 dissolved in a carbonate buffer ( $\mathrm{pH}$ 9.6) at $100 \mathrm{ng} / 100 \mu \mathrm{l}$ per well concentration at $4^{\circ} \mathrm{C}$ overnight. Another recombinant protein prepared in our laboratory with His-tag, recombinant peroxiredoxin2 of $H$. longicornis (HIPrx2), was used as a control antigen (Kusakisako et al., unpublished results). After washing with PBS with $0.05 \%$ Tween 20 (PBS-T), each well was blocked with $150 \mu \mathrm{l}$ of $5 \%$ skimmed milk in PBS-T at $37^{\circ} \mathrm{C}$ for $1 \mathrm{~h}$. The plates were incubated with $100 \mu \mathrm{l} /$ well of rabbit sera in the blocking solution, diluted serially starting at $1: 50$, at $37^{\circ} \mathrm{C}$ for $1 \mathrm{~h}$. ELISA plates were washed several times with PBS-T before applying $100 \mu \mathrm{l}$ of HRP-conjugated polyclonal goat anti-rabbit immunoglobulins (Dako Cytomation, Glostrup, Denmark) in the blocking solution $(1: 2,000$ dilution) in each well and then incubated at $37^{\circ} \mathrm{C}$ for $1 \mathrm{~h}$. After another series of washing, $100 \mu \mathrm{l}$ of TMB One Component HRP Microwell substrate (SurModics, Inc., Eden Prairie, MN, USA) was placed in each well and then incubated at $37^{\circ} \mathrm{C}$ for $30 \mathrm{~min}$. The reaction was stopped by adding $100 \mu \mathrm{l}$ of a mixture of $0.6 \mathrm{~N} \mathrm{H}_{2} \mathrm{SO}_{4}$ and $1 \mathrm{~N} \mathrm{HCl}$ (1:1) in each well. Absorbance was measured using a microplate reader (Bio-Rad, Hercules, CA, USA) at $\mathrm{OD}_{450}$.

\section{Tick infestation and evaluation of vaccination efficacy}

Two weeks after the final immunization, 30 adult female ticks were infested on the ears of each rabbit as described previously [18] until they fed to repletion. After dropping, ticks were weighed and then monitored for survival rate, egg laying and subsequent hatching to larvae as previously described [17]. The effect of immunization was evaluated based on the reduction of the tick's engorged body weight, oviposition, and hatching. Calculations were made using formulas adapted from previous tick vaccination reports [19].

Reduction of tick engorged weight $\left(\mathrm{R}_{\mathrm{W}}\right)=100[1$ (BWV/BWC)], where BWV is the average engorged weight of ticks infested on rHIFER vaccinated rabbits and BWC is the average engorged weight of ticks infested on the control rabbits.

Reduction of oviposition $\left(\mathrm{R}_{\mathrm{O}}\right)=100[1-($ EWV/EWC)], where EWV is the average weight of the eggs from ticks infested on rHIFER vaccinated rabbits and EWC is the average weight of the eggs from ticks infested on the control rabbits.

Reduction on hatching $\left(\mathrm{R}_{\mathrm{H}}\right)=100[1-(\mathrm{AHV} / \mathrm{AHC})]$, where AHV is the percent of ticks with completely hatched eggs from the total number of ticks that laid eggs from rHIFER vaccinated rabbits and AHC is the percent of ticks with completely hatched eggs to the total number of ticks that laid eggs from the control rabbits.

Finally, the overall vaccine efficacy (E) for each group was calculated as $100\left[1-\left(E_{W} \times E_{O} \times E_{H}\right)\right]$, where $E_{W}=B W V /$ BWC, $\mathrm{E}_{\mathrm{O}}=\mathrm{EWV} / \mathrm{EWC}$, and $\mathrm{E}_{\mathrm{H}}=\mathrm{AHV} / \mathrm{AHC}$.

\section{Tick protein preparation and Western blot analyses}

Tick protein samples were prepared from unfed whole adults, partially fed midguts, eggs (20 days after laying), and newly hatched larvae by homogenizing them and suspending in PBS. After sonication, the tick homogenates were centrifuged and the supernatants were collected. The protein concentration of eggs and larvae was determined using Micro BCA kit (Thermo Scientific). To confirm the reactivity of rabbit sera to native tick HIFERs, whole adult and midgut protein samples were separated through SDS-PAGE and then transferred to a polyvinylidene difluoride membrane (Millipore, Bedford, MA, USA). After blocking with $5 \%$ skimmed milk in PBS-T overnight, the membrane was incubated with rabbit sera collected after the third immunization (1:100 dilution) as primary antibodies. Western blot analysis was also performed to further demonstrate the reactivity of rabbit antibodies to rHIFER1, rHIFER2, and rHIPrx2 as a control, with the primary antibodies diluted to 1:3,000. To detect the presence of antibodies in the eggs, recombinant proteins were used as protein samples, and then egg homogenates were used as primary antibodies $(20 \mu \mathrm{g} /$ $500 \mu \mathrm{L}$ ). To demonstrate oxidative stress in eggs and larvae, malondialdehyde (MDA) and protein carbonyl were detected using specific kits for these oxidative stress markers (OxiSelect, Cell Biolabs, Inc., San Diego, CA, USA) as described previously [18]. Tubulin was detected as an internal control using a mouse-derived serum [21]. After incubation with HRP-conjugated goat 
anti-rabbit or anti-mouse immunoglobulin as a secondary antibody (1:30,000 dilution; Dako Cytomation), protein signals were detected using the ECL Prime Western Blotting Detection Reagent (GE Healthcare, Little Chalfont, Buckinghamshire, UK) and images were taken using the FluorChem FC2 Imaging System (Protein Simple, Santa Clara, CA, USA).

\section{Statistical analysis}

The average values of two vaccination trials were calculated and statistical significance was determined using the Student's $t$-test, with significant difference defined by $P<0.05$.

\section{Results}

\section{Purification of recombinant HIFERs and rabbit} immunization

rHIFERs were expressed and purified from E. coli cells. SDS-PAGE showed that the purified rHIFER1 and rHIFER2 have a similar molecular mass of around $26 \mathrm{kDa}$ (Figure 1) as described previously [17]. An antigenspecific ELISA was conducted to monitor the antibody titer of individual rabbits, and then the average antibody titer for each group from the two trials was calculated (Figure 2). Whereas no changes were observed in the antibody titers of the control group, the antibody titers of the groups immunized against rHIFER1 (Figure 2A) and rHlFER2 (Figure 2B) significantly increased after the second immunization. Furthermore, the antibodies also exhibited cross-reactivity to each antigen. rHIFER1immunized rabbits showed an abrupt increase in antibody titer against rHIFER1 after the second immunization, which further increased after the third immunization, while the titer for rHIFER2 only significantly increased after the third immunization. For rHIFER2-immunized rabbits, the antibody titer against rHIFER2 abruptly increased after the second immunization but did not significantly increase further after the third immunization, while the level and the trend of the antibody titer against rHIFER1 were similar to those of the rHIFER1-immunized rabbits. Cross-reactivity of the antibodies was also observed on ELISA using rHlPrx2 as antigen but it was lower than that observed in HlFERs (Additional file 1: Figure S1).

\section{Reactivity of rabbit antibodies to recombinant and native tick HIFERs}

To further evaluate the reactivity of rabbit antibodies, Western blot analysis was performed using rHIFERs, whole tick homogenates, and a midgut homogenate as protein samples. The results showed that sera from immunized rabbits reacted with $\mathrm{rHIFERs}$ and the respective native HIFERs from whole ticks and the midgut (Figure 3). Non-specific bands were seen, particularly on partially-fed midguts, which may be due to the reactivity of secondary

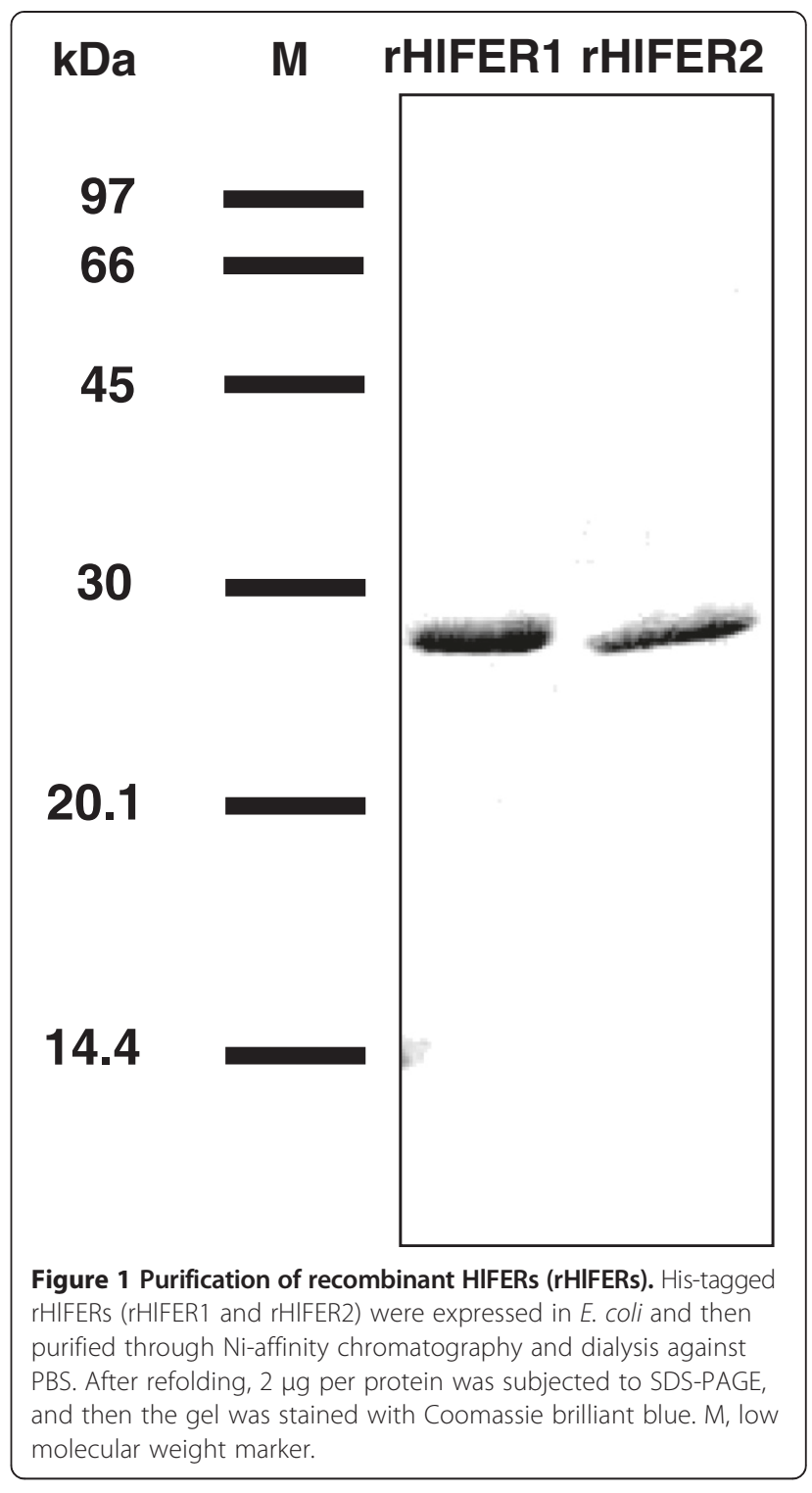

antibody to rabbit blood proteins, since the ticks were fed to rabbits. In addition, cross-reactivity was further demonstrated here, as the sera from the rHIFER1-immunized rabbit showed a positive band against rHIFER2, while the sera from the rHlFER2-immunized rabbit showed a positive band against rHIFER1. Both immune sera also reacted with rHlPrx 2 but to a lesser extent. No positive bands were detected in any of the tested protein samples using sera from control rabbits.

\section{Tick infestation challenge}

The rabbits were infested with adult $H$. longicornis after two weeks from the third immunization. The total number of attached and engorged ticks from rHlFER1-immunized, rHIFER2-immunized, and control rabbits was 78, 80 and 78 , respectively, which was not significantly different. After dropping, the ticks were collected and evaluated for blood 


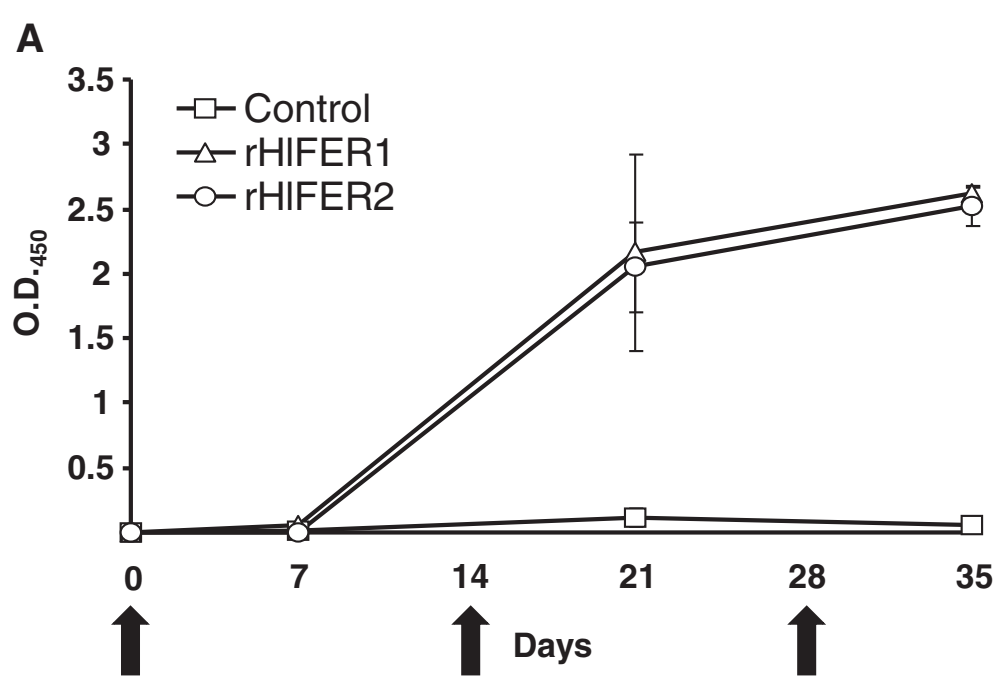

B

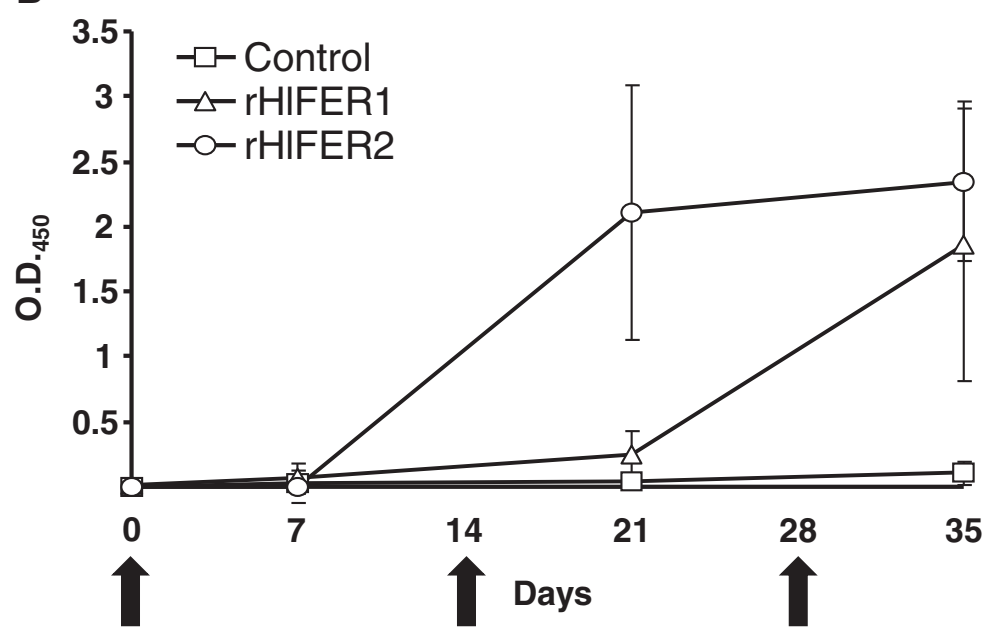

Figure 2 Antigen-specific antibody titer against recombinant HIFER1 (rHIFER1) (A) and recombinant HIFER2 (rHIFER2) (B). Rabbit sera were collected for serum ELISA at days $0,7,21$, and 35. Antibody titers reflect the mean absorbance at $\mathrm{OD}_{450}$ of sera (10 dilution) for each vaccinated group from two separate trials $(n=3)$. The times of immunizations are indicated by arrows. Control, rabbits injected with adjuvant only; rHIFER1, rabbits injected with rHIFER1; rHIFER2, rabbits injected with rHIFER2. Bars represent standard deviation.

feeding and reproduction parameters (Table 1). Ticks infested on rHIFER1- and rHIFER2-immunized rabbits had a lower engorged weight compared to that of the control. However, a significant difference $(P<0.0001)$ was only observed in ticks infested on rHIFER2-immunized rabbits, with a $16 \%$ mean reduction in engorged weight $\left(\mathrm{R}_{\mathrm{W}}\right)$. Moreover, the engorged weight of ticks from rHIFER2-immunized rabbits was also significantly lower $(P=0.0377)$ compared to that of ticks from rHlFER1immunized rabbits. No mortalities were observed in any of the groups until the completion of egg laying. Eggs with abnormal features, such as irregular shape, wrinkled surface and darker colour, were observed from ticks infested on rHIFER2-immunized rabbits (Figure 4) but not from the ticks infested on control and rHlFER1immunized rabbits. Upon completing oviposition, the eggs were weighed and the average egg weight for each group was calculated. Ticks from both rHIFER1- and rHIFER2-immunized rabbits had a significantly lower egg weight $(P<0.05)$. The ticks from rHIFER2-immunized rabbits had the least egg weight among the three groups, significantly lower $(22.4 \%$ reduction, $P<0.0001)$ compared to the control group, but not compared to the rHIFER1 group. The effect of vaccination on hatching was evaluated by the number of ticks with completely hatched eggs. Both rHIFER groups had a reduced number of ticks with completely hatched eggs ( $20 \%$ reduction) compared to the control group. Furthermore, larval 


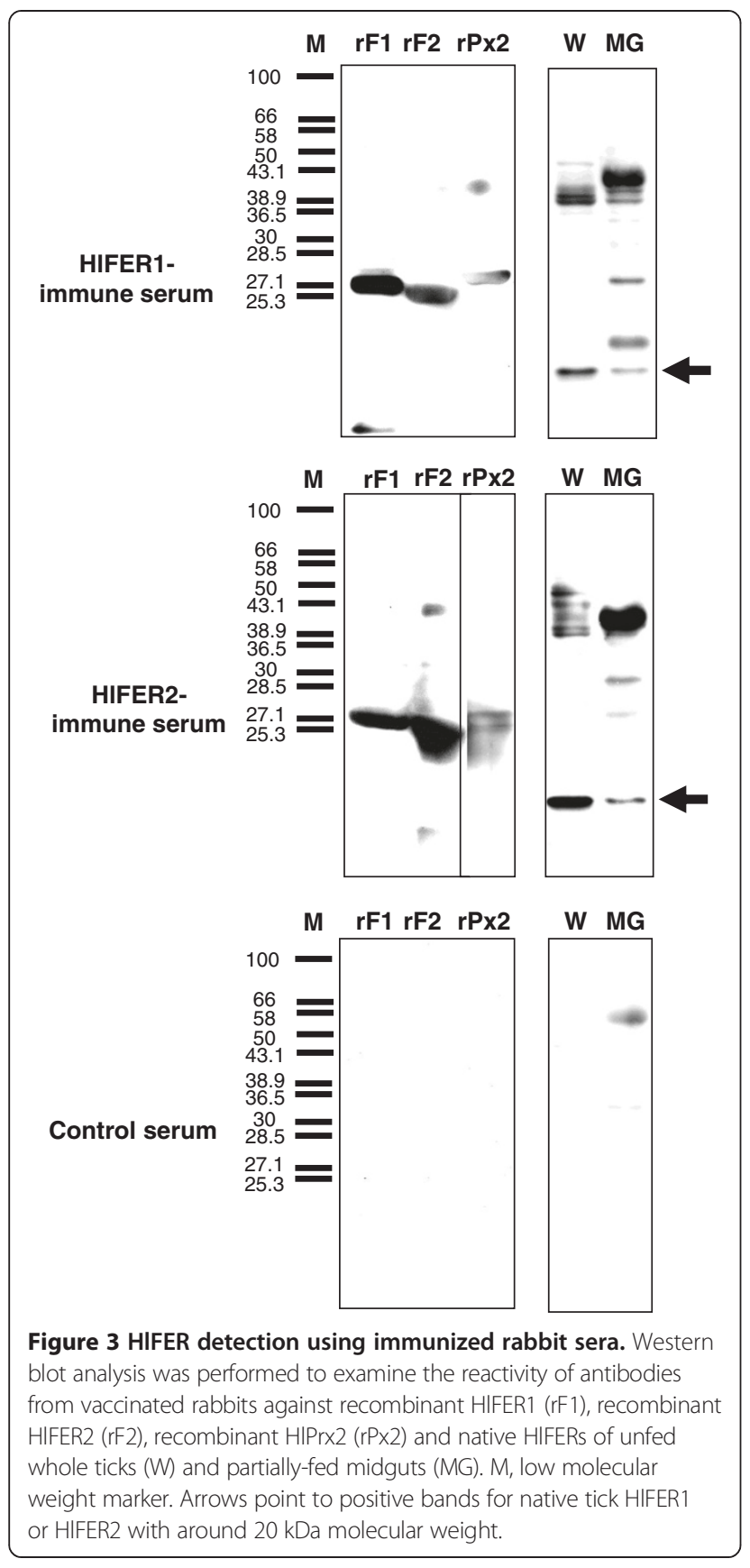

mortality was observed from some ticks in these groups, but not from control. Based on these parameters, the calculated vaccine efficacy (E) for rHIFER1 is $34 \%$ and for rHIFER2 is $49 \%$.

\section{Effects of antibodies on eggs and larvae}

Among the functions of ferritins in ticks is the prevention of iron-mediated oxidative stress [18]. Compared with the previous results of Hlfer gene silencing [17], we observed less pronounced effects on the blood feeding, survival, and egg production of adults. Therefore, we analyzed the eggs and larvae to elucidate the mechanism by which antibodies against HIFERs can affect the ticks. We first detected whether the host antibodies against rHIFERs are present in the eggs (Additional file 2: Figure S2). Western blot analysis showed positive bands for rHIFER1 when egg homogenates from ticks infested on rHIFER1and rHIFER2-immunized rabbits were used as primary antibodies. Meanwhile, a positive band for rHIFER2 was detected when the egg homogenate from ticks infested on rHIFER2-immunized rabbits was used as the primary antibody. No bands were detected when egg homogenates from ticks infested on control rabbit, and secondary antibody alone was used. We then evaluated whether the antibodies can block the function of native HIFER2. Western blot analysis showed that only HIFER2 is present in the eggs and larvae from all groups as we have previously demonstrated [17]. We next detected two oxidative stress biomarkers, MDA from lipid peroxidation and protein carbonyl from protein oxidation [22], using specific antibodies. MDA was detected in the eggs from ticks infested on rHIFER1- and rHIFER2-immunized rabbits and in larvae from ticks infested on rHIFER2immunized rabbits (Figure 4B). Meanwhile, the amount of protein with the carbonyl group was higher in eggs and larvae from ticks infested on rHIFER1- and rHIFER2immunized rabbits than from the control group (Figure 4B). These results suggest the occurrence of oxidative stress in these samples.

\section{Discussion}

The use of vaccines for tick and tick-borne pathogen control has many advantages over the application of

Table 1 Effect of vaccination using recombinant HIFERs on tick feeding and reproduction parameters

\begin{tabular}{|c|c|c|c|c|c|c|c|c|}
\hline Experimental group & $\begin{array}{l}\text { Engorged } \\
\text { weight (mg) }\end{array}$ & $\mathbf{R}_{\mathrm{W}}{ }^{\mathrm{a}}(\%)$ & $\begin{array}{l}\text { Egg } \\
\text { weight (mg) }\end{array}$ & $\mathrm{R}_{\mathrm{O}}{ }^{\mathrm{a}}(\%)$ & $\begin{array}{l}\text { Ticks with } \\
\text { hatched eggs (\%) }\end{array}$ & $\mathbf{R}_{\mathrm{H}}{ }^{\mathrm{a}}(\%)$ & $\begin{array}{l}\text { Ticks with } \\
\text { dead larvae (\%) }\end{array}$ & $\overline{E^{\mathrm{b}}(\%)}$ \\
\hline Adjuvant (control) & $261.2 \pm 51.9$ & 0 & $170.0 \pm 40.4$ & 0 & 100.0 & 0 & 0 & - \\
\hline rHIFER 1 & $243.4 \pm 77.3$ & 6.8 & $142.8 \pm 62.5^{*}$ & 12.4 & $82.0^{*}$ & 18.0 & 5.5 & 34.0 \\
\hline rHIFER2 & $218.0 \pm 66.0^{*}$ & 16.5 & $126.5 \pm 55.8^{*}$ & 22.4 & $80.0^{*}$ & 20.0 & 5.7 & 49.0 \\
\hline
\end{tabular}

Data represent average values from ticks infested on three rabbits for each group, from two separate vaccination trials.

${ }^{a}$ Formulas for the calculation of reductions in engorged weight $\left(R_{W}\right)$, oviposition $\left(R_{O}\right)$, and hatch $\left(R_{H}\right)$ are described in the Methods section

${ }^{b}$ Vaccine efficacy (E) was calculated by comparing tick engorged weight, tick egg weight, and the number of ticks with completely hatched eggs from the recombinant HIFER1 (rHIFER1) or recombinant HIFER2 (rHIFER2) group with those from the control group. The formula is described in the Methods section.

*Significantly different vs. control $(P<0.05$, Student's $t$-test). 


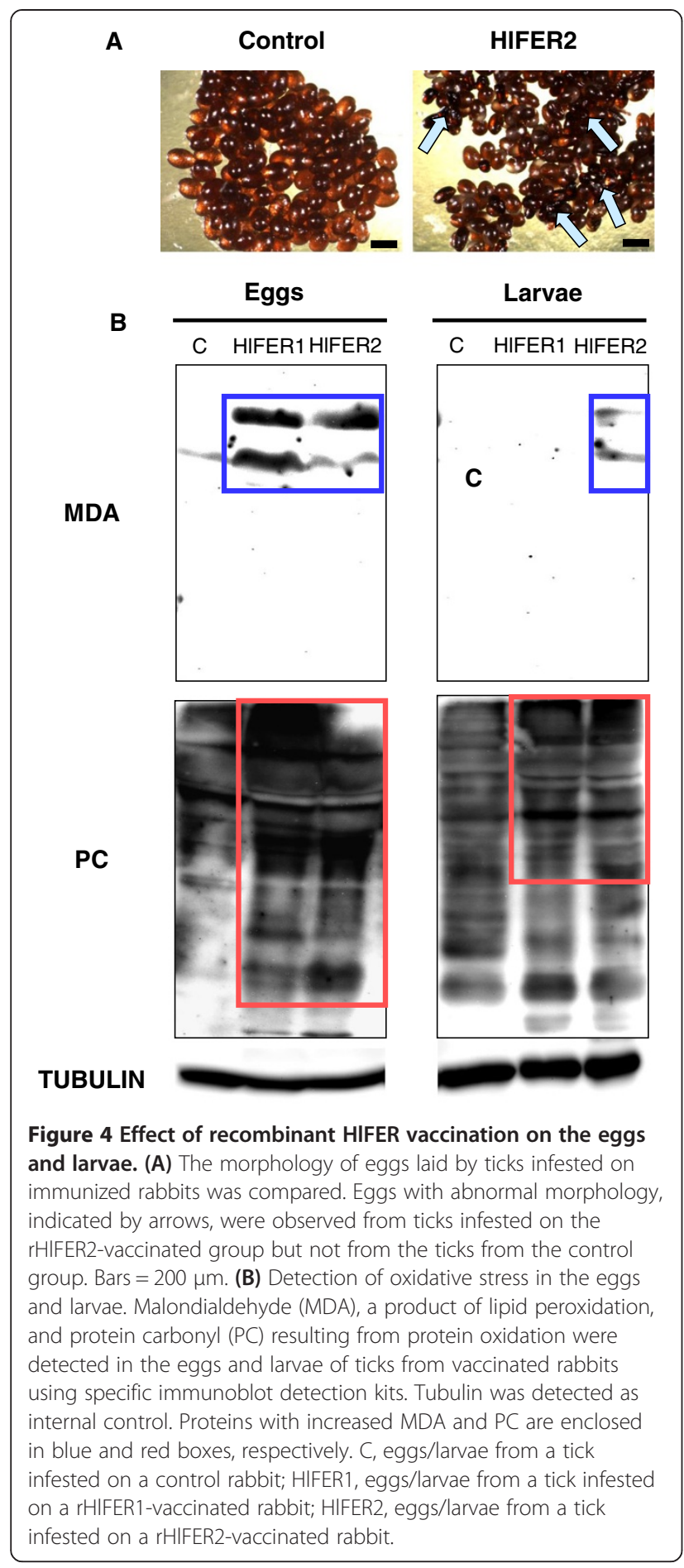

chemical acaricides in terms of resistance development and environmental and animal product contamination concerns. Thus, numerous tick studies are now focused on developing anti-tick vaccines, but most have limited effectiveness in a wide range of tick-species. An anti-tick vaccine affecting multiple tick species and targeting both the ticks and pathogens is still far from reality [13].
Multiple approaches are currently being employed to screen tick antigens and evaluate their potential [12]. Our previous studies on two ferritins of the hard tick $H$. longicornis using RNAi showed that both are physiologically important in blood feeding and reproduction [17] by preventing iron overload and the occurrence of oxidative stress [18]. Furthermore, a previous study showed that FER2 vaccination had considerable effects on infestation of I. ricinus and $R$. microplus [19]. These prompted us to evaluate and compare the potential of two HIFERs as targets for the control of $H$. longicornis.

HIFERs are abundant in different tissues of the tick, with HIFER1 being intracellular in nature, while HIFER2 is secretory [17]. Being concealed antigens, these proteins are not normally encountered by the host immune system during blood feeding, and thus a high level of host antibodies against HIFERs is probably necessary for the blockade of the HIFER function [23]. HIFERs also have complex structures that might require more antibodies binding on them before their function is blocked. The increasing antibody titer after immunization with rHIFERs showed that these proteins are immunogenic. Furthermore, the antibody titer in the immunized rabbits after infestation was as high as the titer after the third immunization, implying that the ticks must have ingested a high amount of anti-rHIFER antibodies during blood feeding. The effects of the antibodies on adult ticks were seen in the reduced engorged bodyweight and oviposition, although these were lower compared to the results of Hlfer gene silencing [17], demonstrating differences in the mechanisms by which RNAi and host antibodies exert their blockade effects against a certain molecule. In contrast to the vaccination result against $I$. ricinus and R. microplus using FER2 [19], no effect on attachment and number of engorging ticks was observed in either group. Nevertheless, the ability of host antibodies to react with native tick HIFERs in the whole tick and midgut shown in Western blot analysis suggests that the antibodies can bind with native HIFERs within the ticks and possibly interfere with their crucial function on blood feeding and egg production. The cross-reactivity of the antibodies to recombinant proteins seen in ELISA and Western blot analyses may be partly attributed to the His-tag, since the antibodies also reacted to HlPrx2. However, since the amino acid sequences of HIFER1 and HIFER2 have $40 \%$ identity, there is still a possibility that the antibodies against tick HIFER1 may cross-react with tick HIFER2, and vice versa.

We wanted to elucidate the mechanism by which antibodies against HIFERs can affect the ticks. It has been demonstrated previously that host antibodies can pass through the midgut barrier of ticks and circulate in the hemolymph [24-26]. Detection of positive bands for recombinant HIFERs on Western blot analysis using egg 
homogenates as primary antibodies may indicate the presence of antibodies against rHIFERs in the eggs of ticks infested on rHIFER-vaccinated rabbits. However, further examination is needed to confirm this result. We also observed eggs with abnormal morphology from ticks infested on recombinant HIFER2 rabbits, similar to our observation after Hlfer 2 gene silencing [17]. Only HIFER2 that comes from the adult ticks is present in the eggs, which may serve to supply iron and/or protection against iron overload during embryonic development [17]. The tick embryo and larvae are normally challenged with oxidative stress as embryogenesis and aging progresses [27]. Therefore, the function of antioxidants, including HIFER2, which keeps iron from promoting the formation of reactive oxygen species, is crucial. While it is unclear whether these anti-HIFER antibodies in the eggs are in the free form or are already bound to HIFER2, its presence and the higher level of oxidative biomarkers in the eggs of the ticks from rHIFER-vaccinated rabbits, indicating the occurrence of oxidative stress, are highly suggestive of interference in native HIFER function, eventually leading to embryonic death and, hence, reduced hatching. Furthermore, the persistence of these antibodies in the larvae after hatching most likely caused oxidative stress and larval mortality. Thus, there is also a possibility that these antibodies may interfere with HIFERs when the larvae feed on a host.

The results of the tick challenge after vaccination showed that rHIFER2 has higher vaccination efficacy than rHIFER1. Although our previous findings suggested that HIFER1 is the major iron-storage HIFER, abundant in most tick tissues, and that silencing the Hlfer 1 gene seemed to have a greater impact on blood feeding, survival and reproduction [17], the importance of HIFER2 as an iron transporter in hard ticks should not be overlooked. Moreover, the mainly intracellular localization of HIFER1 might have made it inaccessible to anti-HIFER1 antibodies, with the exception of the midgut, where the antibody may interact with HIFER1 within digestive cells. On the other hand, HIFER2, being a secretory protein, may be more accessible to the antibodies after passing through the midgut barrier. HIFER2 is abundant in the hemolymph, circulating within the tick's body, and as mentioned earlier, can be passed to the eggs. This systemic function of HIFER2, as well as its exclusive presence in the eggs, may have contributed to its higher vaccine efficacy. Furthermore, the antibodies against HIFER2 showed a higher cross-reactivity compared to antibodies against HIFER1.

Ferritin is a highly conserved molecule among different tick species that is ubiquitous in most tick tissues and in all developmental stages. HIFERs have high homology with other tick ferritins. This makes tick ferritins a highly preferable candidate target antigen for the formulation of a multi-species anti-tick vaccine [13]. In contrast to
HIFER1, HIFER2 has a lower identity/similarity, less than $40 \%$, to vertebrate ferritins [17]. The secretory FER2 is also considered unique to ticks [16]. In the light of the results in this study, HIFER2 is a better antigen than HIFER1, supporting the previous vaccination study in other tick species [19].

\section{Conclusion}

The importance of ticks as ectoparasites of humans and animals and vectors of several diseases is widely known. Vaccination is highly anticipated to overcome the drawbacks of chemical acaricide control. Thus, numerous tick studies are aiming to identify a single or multiple target antigens that can affect multiple tick species and, ideally, also target tick-borne pathogens $[1,12,13]$. Most of the tick vaccination studies focus on controlling Rhipicephalus and Ixodes species. Here we investigated and compared the potential of two HIFERs as targets for the control of the hard tick $H$. longicornis. RNAi is a good technique for screening candidate antigens for vaccine development [12]. Our previous gene silencing studies showed that HIFERs are crucial to the blood feeding and reproduction of $H$. longicornis [17], providing protection against ironmediated oxidative stress [18] and making them good candidate vaccine antigens. Our vaccination experiments showed that both rHIFER1 and rHIFER2 are highly immunogenic, inducing host antibody production. The tick infestation challenge showed that immunizing the host with rHIFER2 significantly reduced the engorged weight of the infested ticks. Immunization with either of the rHIFERs reduced the number of eggs and the number of ticks with completely hatched eggs, with rHIFER2 producing a greater reduction. Based on these tick parameters, rHIFER2 showed a higher vaccine efficacy of almost $50 \%$. Moreover, eggs with abnormal morphology were observed from ticks infested on rHIFER2-immunized rabbits. We also attempted to elucidate the mechanism of anti-HlFER antibody protection against ticks. Induced antibodies against rHIFERs were detected in the eggs. The presence of a higher level of molecules produced during lipid and protein oxidation in the eggs and larvae from ticks infested on rHIFER-vaccinated rabbits indicates the occurrence of oxidative stress, suggesting that the antibodies interfered with the HIFER2 function. Collectively, our results show that the secretory HIFER2 is a good target for the control of $H$. longicornis, supporting the findings of a previous study targeting FER2 in other hard tick species [19]. While its vaccine efficacy may still be lower compared with those of other studied vaccine antigens, inclusion of HIFER2 in the vaccine with other antigens may yield better results than immunizing with a single kind of antigen and may provide multi-species protection since FER is a highly conserved molecule. 


\section{Additional files}

Additional file 1: Figure S1. ELISA using recombinant $H$. longicornis peroxiredoxin (HIPrx2) as antigen. Sera collected on days 0, 7, 21 and 35 from recombinant HIFER1 (rHIFER1), recombinant HIFER2 (rHIFER2) and Control rabbits were checked for reactivity against $r$ HIPrX2.

Additional file 2: Figure S2. Detection of antibodies in the eggs of ticks infested on vaccinated rabbits. (A) Induced antibodies from immunized rabbits were detected in the eggs by Western blot analysis using recombinant HIFER1 (rHIFER1) and recombinant HIFER2 (rHIFER2) as protein samples. Egg homogenates from ticks infested on the control (C), rHIFER1- (HIFER1), and rHIFER2- (HIFER2) vaccinated rabbits were used as primary antibodies. Polyclonal HRP-conjugated goat anti-rabbit immunoglobulins were used as secondary antibodies to detect rabbit antibodies. Arrows point to positive bands for rHIFER1 or rHIFER2. (B) As an additional control, the membrane was incubated with polyclonal HRP-conjugated goat anti-rabbit immunoglobulins only.

\section{Abbreviations}

ELISA: Enzyme-linked immunosorbent assay; FER: Ferritin;

HIFER1: Haemaphysalis longicornis ferritin 1 (intracellular); HIFER2: H. longicornis ferritin2 (secretory); HRP: Horseradish peroxidise; IPTG: Isopropyl B-D-1-thiogalactopyranoside; MDA: Malondialdehyde; ORF: Open reading frame; PBS: Phosphate-buffered saline; PBS-T: PBS with 0.05\% Tween 20; PC: Protein carbonyl; rHIFERs: Recombinant H. longicornis ferritins; RNAi: RNA interference; $R_{W}$ : Reduction of tick engorged weight; Ro: Reduction of oviposition; $R_{H}$ : Reduction of hatching; SDS-PAGE: Sodium dodecyl sulphate polyacrylamide gel electrophoresis; SFTS: Severe fever with thrombocytopenia syndrome.

\section{Competing interests}

The authors declare that they have no competing interests.

\section{Authors' contributions}

RLG, TM and TT designed the study; RLG, HM and KK performed the experiments; RLG, TM, RUS, NT, MM, FF and TT analyzed the data; RLG wrote the manuscript. All authors read and approved the final manuscript.

\section{Acknowledgments}

This study was supported by a cooperative research grant (26-joint-6) of the National Research Center for Protozoan Diseases, Obihiro University of Agriculture and Veterinary Medicine, Japan Society for the Promotion of Science (JSPS) KAKENHI Grant Numbers 25252050-1, 25292173 and 26660229, and Morinaga Foundation. The first-named author is supported by the Japanese Government Ministry of Education, Culture, Sports, Science, and Technology Scholarship (Monbukagakusho: MEXT) for doctoral fellowship.

\section{Author details}

${ }^{1}$ Department of Pathological and Preventive Veterinary Science, The United Graduate School of Veterinary Science, Yamaguchi University, Yoshida, Yamaguchi 753-8515, Japan. ${ }^{2}$ Laboratory of Infectious Diseases, Joint Faculty of Veterinary Medicine, Kagoshima University, 1-21-24 Korimoto, Kagoshima 890-0065, Japan. ${ }^{3}$ Laboratory of Food Chemistry, Department of Biochemistry and Biotechnology, Division of Molecular Functions of Food, Faculty of Agriculture, Kagoshima University, 1-21-24 Korimoto, Kagoshima 890-0065, Japan. ${ }^{4}$ National Research Center for Protozoan Diseases, Obihiro University of Agriculture and Veterinary Medicine, Inada-cho, Obihiro, Hokkaido 080-8555, Japan. ${ }^{5}$ Department of Parasitology, Kitasato University School of Medicine, Kanagawa 252-0374, Japan. ${ }^{6}$ National Agricultural and Food Research Organization, 3-1-5 Kannondai, Tsukuba, Ibaraki 305-0856, Japan.

Received: 10 July 2014 Accepted: 7 October 2014

Published online: 12 October 2014

\section{References}

1. Sprong H, Trentelman J, Seemann I, Grubhoffer L, Rego ROM, Hajdusek O, Kopacek P, Sima R, Nijhof AM, Anguita J, Winter P, Rotter B, Havlikova S, Klempa B, Schetters TP, Hovius JWR: ANTIDote: anti-tick vaccines to prevent tick-borne diseases in Europe. Parasit Vectors 2014, 7:77.
2. Marcelino I, de Almeida AM, Ventosa M, Pruneau L, Meyer DF, Martinez D, Lefrancois T, Vachiery N, Coelho AV: Tick-borne diseases in cattle: applications of proteomics to develop new generation vaccines. J Proteomics 2012, 75:4232-4250.

3. Heath ACG: Vector competence of Haemaphysalis longicornis with particular reference to blood parasites. Surveillance 2002, 29:12-14.

4. Jonjegan F, Uilenberg G: The global importance of ticks. Parasitology 2004, 129:S3-S14.

5. Liu S, Chengliang C, Wang C, Amer S, Lv H, He H, Sun J, Lin J: Systematic review of severe fever with thrombocytopenia syndrome: virology, epidemiology, and clinical characteristics. Rev Med Virol 2014, 24:90-102.

6. Takahashi T, Maeda K, Suzuki T, Ishido A, Shigeoka T, Tominaga T, Kamei T, Honda M, Ninomiya D, Sakai T, Senba T, Kaneyuki S, Sakaguchi S, Satoh A, Hosokawa T, Kawabe Y, Kurihara S, Izumikawa K, Kohno S, Azuma T, Suemori K, Yasukawa M, Mizutani T, Omatsu T, Katayama Y, Miyahara M, ljuin M, Doi K, Okuda M, Umeki K, et al: The first identification and retrospective study of severe fever with thrombocytopenia syndrome in Japan. $J$ Infect Dis 2014, 6:816-827.

7. Chang MS, Woo JH: Severe fever with thrombocytopenia syndrome: tick-mediated viral disease. J Korean Med Sci 2013, 28:795-796.

8. de la Fuente J, Kocan KM: Strategies for development of vaccines for control of ixodid tick species. Parasite Immunol 2006, 28:275-283.

9. Merino $O$, Alberdi $P$, de la Lastra JM P, de la Fuente J: Tick vaccines and the control of tick-borne pathogens. Front Cell Infect Microbiol 2013, 3:30.

10. Willadsen P, Bird P, Cobon GS, Hungerford J: Commercialisation of a recombinant vaccine against Boophilus microplus. Parasiol 1995, 110:43-50.

11. Willadsen P: Tick control: thoughts on a research agenda. Vet Parasitol 2006, 138:161-168.

12. de la Fuente J, Merino O: Vaccinomics, the new road to tick vaccines. Vaccine 2013, 31:5923-5929.

13. Parizi LF, Githaka NW, Logullo C, Konnai S, Masuda A, Ohashi K, da Silva Vl: The quest for a universal vaccine against ticks: Cross-immunity insights. Vet J 2012, 194:158-165.

14. Antunes S, Merino O, Mosqueda J, Moreno-Cid JA, Bell-Sakyi L, Fragkoudis R, Weisheit S, de la Lastra JMP, Alberdi P, Domingos A, de la Fuente J: Tick capillary feeding for the study of proteins involved in tick-pathogen interactions as potential antigens for the control of tick infestation and pathogen infection. Parasit Vectors 2014, 7:42.

15. Arosio P, Ingrassia R, Cavadini P: Ferritins: A family of molecules for iron storage, antioxidation and more. Biochim Biophys Acta 2009, 1790:589-599.

16. Hajdusek O, Sojka D, Kopacek P, Buresova V, Franta Z, Sauman I, Winzerling J, Grubhoffer L: Knockdown of proteins involved in iron metabolism limits tick reproduction and development. Proc Natl Acad Sci U S A 2009, 106:1033-1038.

17. Galay RL, Aung KM, Umemiya-Shirafuji R, Maeda H, Matsuo T, Kawaguchi H, Miyoshi N, Suzuki H, Xuan X, Mochizuki M, Fujisaki K, Tanaka T: Multiple ferritins are vital to successful blood feeding and reproduction of the hard tick Haemaphysalis longicornis. J Exp Biol 2013, 216:1905-1915.

18. Galay RL, Umemiya-Shirafuji R, Bacolod ET, Maeda H, Kusakisako K, Koyama J, Tsuji N, Mochizuki M, Fujisaki K, Tanaka T: Two kinds of ferritin protect ixodid ticks from iron overload and consequent oxidative stress. PLoS ONE 2014, 9:e90661.

19. Hajdusek O, Almazan C, Loosova G, Villar M, Canales M, Grubhoffer L, Kopacek P, de la Fuente J: Characterization of ferritin 2 for the control of tick infestations. Vaccine 2010, 28:2993-2998.

20. Fujisaki K: Development of acquired resistance and precipitating antibody in rabbits experimentally infested with females of Haemaphysalis longicornis (Ixodoidea: Ixodidae). Natl Inst Anim Health $Q$ 1978, 18:27-38.

21. Umemiya-Shirafuji $R$, Tanaka T, Boldbaatar D, Tanaka T, Fujisaki K: Akt is an essential player in regulating cell/organ growth at the adult stage in the hard tick Haemaphysalis longicornis. Insect Biochem Mol Biol 2012, 42:164-173.

22. Valko M, Rhodes CJ, Moncol J, Izakovic M, Mazur M: Free radicals, metals and antioxidants in oxidative-stress induced cancer. Chem Biol Interact 2006, 160:1-40.

23. Nuttall PA, Trimnell AR, Kazimirova M, Labuda M: Exposed and concealed antigens as vaccine targets for controlling ticks and tick-borne diseases. Parasite Immunol 2006, 28:155-163.

24. Jasinkas A, Jaworski DC, Barbour AG: Amblyomma americanum: specific uptake of immunoglobulins into tick hemolymph during feeding. Exp Parasitol 2000, 96:213-221. 
25. Ackerman S, Clare FB, McGill TW, Sonenshine DE: Passage of host serum components, including antibody, across the digestive tract of Dermacentor variabilis (Say). J Parasitol 1981, 67:737-740.

26. Fujisaki K, Kamio T, Kitaoka S: Passage of host serum components, including antibodies against Theileria sergenti, across the digestive tract of argasid and ixodid ticks. Ann Trop Med Parasitol 1984, 78:449-450.

27. Freitas D, Rosa R, Moraes J, Campos E, Logullo C, Da Silva Vaz Jr I, Masuda A: Relationship between glutathione S-transferase, catalase, oxygen consumption, lipid peroxidation and oxidative stress in eggs and larvae of Boophilus microplus (Acarina: Ixodidae). Comp Biochem Physiol A 2007, 146:688-694.

doi:10.1186/s13071-014-0482-x

Cite this article as: Galay et al.: Evaluation and comparison of the potential of two ferritins as anti-tick vaccines against Haemaphysalis longicornis. Parasites \& Vectors 2014 7:482.

\section{Submit your next manuscript to BioMed Central and take full advantage of:}

- Convenient online submission

- Thorough peer review

- No space constraints or color figure charges

- Immediate publication on acceptance

- Inclusion in PubMed, CAS, Scopus and Google Scholar

- Research which is freely available for redistribution 